\title{
Erlotinib and Concurrent Chemoradiation in Pretreated NSCLC Patients: Radiobiological Basis and Clinical Results
}

\author{
Sara Ramella, ${ }^{1}$ Antonio Maria Alberti, ${ }^{2}$ Eugenio Cammilluzzi, ${ }^{2}$ Michele Fiore, ${ }^{1}$ \\ Edy Ippolito, ${ }^{1}$ Carlo Greco, ${ }^{1}$ Angelo Luca De Quarto, ${ }^{2}$ Sara Ramponi, ${ }^{2}$ Giovanni Apolone, ${ }^{3}$ \\ Lucio Trodella, ${ }^{1}$ Alfredo Cesario, ${ }^{4,5}$ and Rolando Maria D'Angelillo ${ }^{1}$ \\ ${ }^{1}$ Radiation Oncology, Campus Bio-Medico University, Via Álvaro del Portillo 21, 00128 Roma, Italy \\ ${ }^{2}$ Medical Oncology, Sandro Pertini Hospital, Via dei Monti Tiburtini 385, 00157 Roma, Italy \\ ${ }^{3}$ Scientific Directorate, IRCCS-Arcispedale Santa Maria Nuova, Viale Risorgimento 80, 42123 Reggio Emilia, Italy \\ ${ }^{4}$ Thoracic Surgery, Catholic University, Largo Agostino Gemelli 8, 00168 Roma, Italy \\ ${ }^{5}$ Deputy Scientific Director, IRCCS San Raffaele Pisana, Via della Pisana 235, 00163 Roma, Italy
}

Correspondence should be addressed to Sara Ramella; s.ramella@unicampus.it

Received 30 April 2013; Revised 8 July 2013; Accepted 15 July 2013

Academic Editor: Joanna Domagala-Kulawik

Copyright (c) 2013 Sara Ramella et al. This is an open access article distributed under the Creative Commons Attribution License, which permits unrestricted use, distribution, and reproduction in any medium, provided the original work is properly cited.

\begin{abstract}
Aims. To establish feasibility of the combination of Erlotinib and concurrent chemoradiation in pre-treated patients with locally advanced or metastatic NSCLC. Materials and Methods. Data regarding 60 consecutive patients with NSCLC previously treated with chemotherapy alone were prospectically collected. All patients started Erlotinib concurrently with chemotherapy and radiation delivered to primary tumor. These data were retrospectively analyzed (observational study). Feasibility and toxicity were the primary endpoints, with response rate and progression being the secondary ones, while survival data are reported just as exploratory analysis. The EGFR mutational status was recorded in $32 \%$ of cases and it was always wild type. Results. Compliance to the combination protocol was good. Grade 3-4 esophagitis and acute lung toxicity occurred in $2 \%$ and $8 \%$ of patients, respectively. No progressive disease was recorded in the majority of cases (65\%). Median OS and PFS were 23.3 and 4.7 months, respectively. Patients not responding to chemotherapy administered prior to chemoradiation achieved an objective response rate of $53.3 \%$ and complete response in $13.3 \%$ of cases. Conclusions. The addition of Erlotinib to chemoradiation in inoperable NSCLCs is feasible with interesting efficacy profile. These preliminary results warrant further investigation in patients with locally advanced nonmetastatic NSCLC with EGFR mutations.
\end{abstract}

\section{Introduction}

The overexpression of EGFR plays a key role in cellular proliferation, metastasis, apoptosis inhibition, and chemoradioresistance [1]; its targeting with tyrosine kinase inhibitors (TKIs) has improved survival in metastatic NSCLC patients [2]. The standard treatment for locally advanced inoperable NSCLC is concurrent chemoradiation. With modern techniques, it guarantees effective and safe treatment [3]. Experimental evidence suggests that the TKIs could have a radiosensitizing effect [4]. Many mechanisms of this radiosensitizing effect have been proposed and in vivo studies have confirmed synergistic growth inhibition of radiation and TKIs [5]. In
NSCLC models Erlotinib clearly enhances radiation cytotoxicity [6]. Radiobiological criteria and basis of evidence have led to investigating the role of this combination at several levels.

(i) Radiosensitization of the cancer cell by altering intracellular signaling: as previously reported, the overexpression of EGFR in solid tumors is correlated with increased radioresistance. This is apparently due, in first instance, to the ability of radiation to interact with EGFR causing receptor activation even in absence of EGF, for instance by TGF $\alpha$ release and EGFR autophosphorylation increase. This activation 
triggers intracellular pathways cascade, mainly via the RAS/RAF/MAP kinases (resulting in proliferative stimulus) and via the PI3K/AKT one (resulting in the inhibition of the apoptosis). This effect has been proposed to represent a central mechanism for accelerated cellular repopulation during radiation treatment [7].

(ii) Cell cycle kinetics: Erlotinib, when combined with radiation, has been demonstrated to be able to promote a reduction in the $\mathrm{S}$-phase fraction (which is the most radioresistant cell cycle phase), inducing accumulation of cells in G1 and G2 [8].

(iii) Apoptosis: Erlotinib and radiation induced an increase in apoptosis as determined by caspase activity. Poly(ADP-ribose) polymerase (PARP) cleavage increases when Erlotinib is combined with radiation [8].

(iv) DNA repair: Erlotinib attenuates radiation induced expression of DNA repair protein [8]. The main action of radiation is killing cancer cells by DNA damage. When radiation reaches cell surface causes EGFR internalization. The receptor moves into the nucleus by binding proteins (Ku70/Ku80 and DNA-PKcs) and activates damage repair. If EGFR is blocked by antibodies or TKIs, the complex does not enter the nucleus resulting in inhibition of DNA repair. Rad51 is a repair protein which represents a central part of the homologous recombination process during the DNA repair and Erlotinib attenuates the increase of Rad51 after radiation exposure [9]. The potential influence of Erlotinib on the DNA damage repair is amplified in vivo respect to in vitro setting because of the delivering of multiple versus single fractions of radiation [8].

(v) Clonogenic cells: Erlotinib influences cancer cell clonogenic survival, with a modest but consistent reduction in clonogenic survival when the TKI is administered before the radiation treatment [9].

To the best of our knowledge, only sporadic reports exist in the literature about clinical experiences adopting this combination which refer to small populations treated with small molecules plus radiation, with or without concurrent chemotherapy [10-12].

Currently, Erlotinib has a well-established role in firstand second-line treatment but few data are still present in locally advanced NSCLC while radiotherapy has shown an important action against symptoms onset. Sometimes, in daily clinical practice, patients treated with upfront chemotherapy experienced minimal response with stable disease or partial response with tumor reduction among 30\% in volume and are candidates for palliative chemoradiation or second line Erlotinib. The aim of this study was twofold: to report feasibility (defined as compliance to the protocol) and activity of combining both strategies (Erlotinib plus chemoradiation) in this poor prognostic group.

\section{Materials and Methods}

We treated patients with locally advanced (IIIA-IIIB) NSCLC (excluding those with cytologically confirmed malignant pleural or pericardial effusion) or mediastinal recurrences after surgery or oligometastatic disease (up to two sites of distant metastasis) with good performance status and adequate blood marrow reserve. All patients received upfront chemotherapy and those classified as nonresponders or minimal responders (among 30\% in reduction of tumor volume) went on to have Erlotinib $150 \mathrm{mg}$ PO daily, delivered concurrently with chemoradiation.

Induction chemotherapy consisted in 4-6 cycles of chemotherapy according to patient's tolerance and the revaluation CT was performed about 1 month after the last chemotherapy cycle.

Radiotherapy was delivered using a linear accelerator (CLINAC C2100, Varian) with a 6-15 MV photon beam up to a median dose of $59.4 \mathrm{~Gy}$ and $1.8 \mathrm{~Gy}$ as daily fractionation. All patients underwent 3D-treatment planning and were immobilized by customized devices. Radiotherapy was administered with an angled fields technique (planar and no planar) [13] to include the Planning Target Volume (PTV) in the $95-107 \%$ isodose area. The Gross Tumor Volume (GTV) was defined as tumor extension and metastatic lymph nodes CT and/or PET 18FDG defined. The Clinical Target Volume was equal to GTV, and the PTV consisted of GTV plus $1 \mathrm{~cm}$ margin. Elective Nodal Irradiation was never used. The dosevolume constraints for total lung were set as follows: V20 $\leq$ $31 \%$, V30 $\leq 18 \%$, and MLD $\leq 20 \mathrm{~Gy}$; for the ipsilateral lung $\mathrm{V} 20 \leq 52 \%$ and V30 $\leq 39 \%$; the maximum spinal cord dose was $38 \mathrm{~Gy}$; for the esophagus V50<30\%, and for the heart $\mathrm{V} 40<50 \%$.

The total dose of 59.4 Gy was delivered in 33 fractions/ 6.5 weeks of treatment duration. Seven weekly gemcitabine (350 mg/mq) and two pemetrexed cycles $(500 \mathrm{mg} / \mathrm{mq})$ were administered concurrently to radiotherapy.

Toxicity was recorded according to the Common Toxicity Criteria scale 3.0 (CTC-AE). When grade 2 or 3 esophageal, pulmonary, and cardiac toxicity or grade 3-4 hematological and skin toxicity appeared, radiation was interrupted and restarted upon resolution. Administration of chemotherapy was delayed in case of grade 2 hematological toxicity, while a $25 \%$ dose reduction was applied if grade 3 hematological toxicity appeared. Radiotherapy was discontinued in case of grade 4 nonhematological toxicity or persistent grade 3 nonhematological toxicity with symptoms not recovered after 14 days with specific therapy. Chemotherapy was discontinued in the case of grade 4 hematological toxicity.

Response evaluation was performed using the Response Evaluation Criteria in Solid Tumor (RECIST) by CT scan 4-5 weeks after the end of the treatment. 18 FDG PET/TC was performed after 4-6 months after the end of radiotherapy, then annually or for clinical suspicious.

Pyrosequencing was performed on material obtained from histological assessment, to verify the sequencing data of the hotspots of EGFR (exons 18-19-21) and to assess the proportion of mutant alleles in microdissected specimens using a Pyrosequencing PSQ 96MA (Pyrosequencing, Uppsala, 
Sweden). Patients underwent a monthly followup for the first 3 months every 3 months for the following 2 years, and every 4-6 months for the next 3 years.

2.1. Statistical Analysis. Primary endpoints were feasibility and toxicity. The secondary endpoints included response rate and progression. Overall survival (OS; defined as the time between diagnosis and event or last visit) and progressionfree survival (PFS; defined as the time between the end of chemoradiation and disease progression) were reported only as exploratory data. Statistical analysis was performed with SYSTAT, ver. 11.0 (SPSS, Chicago, IL).

\section{Results}

3.1. Patients Characteristics. Between July 2007 and May 2010, 60 consecutive patients were observed and treated. Patient's characteristics are listed in Table 1. Median age was 65 years (range $39-83$ ), with male prevalence (70\%). Twenty patients had a stage IIIA disease (33\%), 19 had stage IIIB (32\%), 6 had mediastinal recurrences after surgery (10\%), and 15 patients had oligometastatic disease (25\%). Among metastatic patients, 14 had one distant metastasis while the last one had one lung and one brain metastases (total distant metastases: 16). Histology was nonsquamous in $65 \%$ of cases, squamous in $33 \%$, and not otherwise specified in 1 patient $(2 \%)$.

Fift-four patients (90\%) received upfront chemotherapy, whilst the remaining 6 patients were postsurgery, with median number of 4 courses (range 1-12). In 28 patients (52\%) chemotherapy obtained a stable disease, while 24 and 2 patients had a minimal partial response and a progression disease, respectively.

3.2. Toxicity. Table 2 summarizes treatment related toxicities. Only one patient was unable to complete the combined treatment as planned due to toxicity (pulmonary), while two patients stopped treatment due to tumor progression and one due to a fall in performance status. Four patients (7\%) developed a grade 3 skin rash requiring dose reduction of Erlotinib to $100 \mathrm{mg}$ daily. Grade 3 diarrhea occurred in 2 patients while nausea, mucositis, and photophobia were reported in a few patients and always classified as grade 1-2 toxicity. Grade 34 esophagitis and pulmonary toxicity occurred in $2 \%$ and $8 \%$ of patients, respectively. After treatment end, two patients experienced a fatal pulmonary toxicity: one experienced radiation pneumonitis associated with multiple pulmonary metastasis, while the other one developed sepsis with positive hemoculture for Staphylococcus epidermidis. Subacute and late pulmonary toxicity was recorded in $23 \%$ of patients as radiographic changes not requiring oxygen therapy. Finally, grade 3-4 hematological toxicity was recorded as follows: thrombocytopenia $12 \%$; leucopenia $30 \%$; anemia $5 \%$. Liver toxicity, recorded as modification in liver enzymes, occurred as grade 3-4 toxicity in 8 patients, requiring Erlotinib and chemotherapy end.

Complete or partial response, and stable disease were achieved in 6,18 , and 15 patients, respectively, accounting for an overall $65 \%$ of "no progressive disease." Three patients
TABLE 1: Patient characteristics.

\begin{tabular}{|c|c|}
\hline & Total \\
\hline$N$ & 60 \\
\hline Age (yr), median (range) & $65.5(39-83)$ \\
\hline \multicolumn{2}{|l|}{ Sex, $N(\%)$} \\
\hline Male & $42(70)$ \\
\hline Female & $18(30)$ \\
\hline \multicolumn{2}{|c|}{ ECOG performance status, $N(\%)$} \\
\hline 0 & $45(75)$ \\
\hline 1 & $15(25)$ \\
\hline \multicolumn{2}{|l|}{ Stage, $N(\%)$} \\
\hline IIIA & $20(33)$ \\
\hline IIIB & $19(32)$ \\
\hline Relapse (mediastinal) & $6(10)$ \\
\hline IV (oligometastatic) & $15(25)$ \\
\hline \multicolumn{2}{|l|}{ Histology, $N(\%)$} \\
\hline Squamous cell & $20(33)$ \\
\hline Adenocarcinoma & $39(65)$ \\
\hline NSCLC NOS & $1(2)$ \\
\hline \multicolumn{2}{|c|}{ Previous chemotherapy, $N(\%)$} \\
\hline Yes & $54(90)$ \\
\hline No & $6(10)$ \\
\hline \multicolumn{2}{|c|}{ Number of previous chemotherapy cycles: } \\
\hline Median (range) & $4(1-12)$ \\
\hline \multicolumn{2}{|c|}{ Response to previous chemotherapy } \\
\hline PR & $24(40)$ \\
\hline SD & $28(46)$ \\
\hline $\mathrm{PD}$ & $2(4)$ \\
\hline No previous chemo & $6(10)$ \\
\hline
\end{tabular}

Legend: PR: partial response; SD: stable disease; PD: progression disease.

TABLe 2: Treatment related toxicity.

\begin{tabular}{lcc}
\hline & Grade 1-2 & Grade 3-4 \\
\hline Anemia & Hematological & \\
White blood cell & $26(43 \%)$ & $3(5 \%)$ \\
Platelet & $30(50 \%)$ & $18(30 \%)$ \\
\hline & Nonhematological & $7(12 \%)$ \\
Esophagitis & $32(55 \%)$ & $1(2 \%)$ \\
Pulmonary & $14(23 \%)$ & $5(8 \%)$ \\
Rush & $39(65 \%)$ & $4(7 \%)$ \\
Diarrhea & $10(17 \%)$ & $3(5 \%)$ \\
Nausea & $9(15 \%)$ & - \\
Photophobia & $5(8 \%)$ & - \\
Hepatic enzymes & $30(50 \%)$ & - \\
Mucositis & $12(20 \%)$ & $(13 \%)$ \\
\hline
\end{tabular}

had an in-field progression (5\%), 1 had local and distant relapse, and 17 showed distant relapse (28\%). Patients who are "no responders" to upfront chemotherapy, achieved an objective response rate of $53.3 \%$ after chemoradiation and 


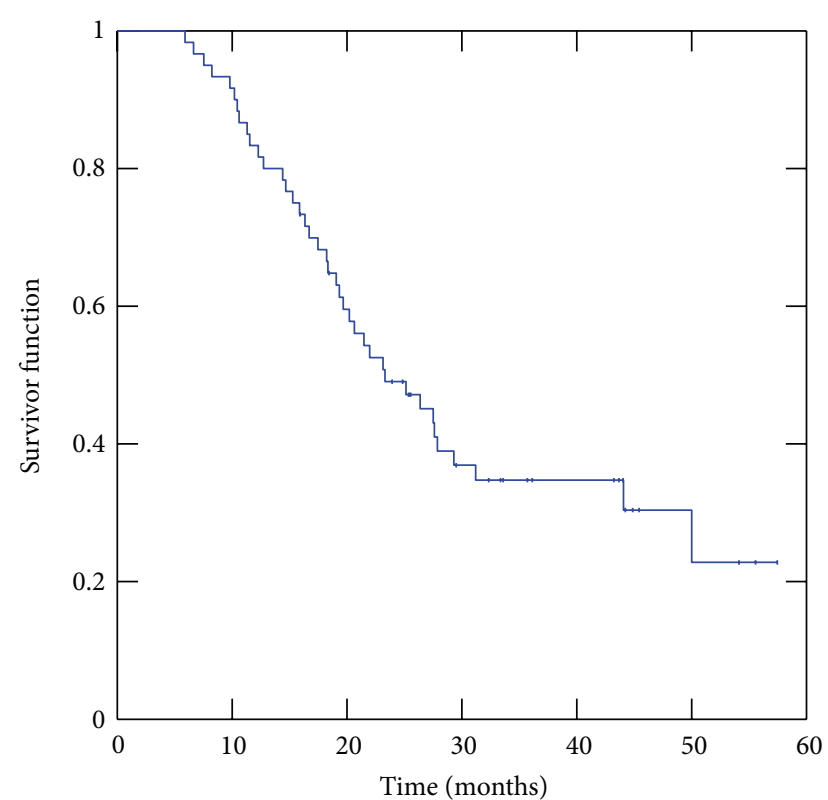

FIGURE 1: Overall survival for the whole group.

TKIs combination with "complete response" in 13.3\% of cases. No particular association was noted between skin rash or histology with clinical response. Survival median survival was 23.3 months (Figure 1). One-, 2-, and 3-year overall survival was $81 \%, 46 \%$, and $29 \%$, respectively.

As expected, patients with complete/partial response experienced a better survival (44 months) than no-responder ones (23.3 months) or those with progressive disease (17.5 months; $P=0.025$ ) (Figure 2). With a median followup from the end of concomitant therapy of 33.7 months, we have recorded 39 deaths, while 14 patients are still alive with residual tumor and 5 patients are free from any tumor evidence. Analysis of the EGFR mutation status was performed in 19/60 patients (32\%); none of these had EGFR mutations.

\section{Discussion}

Our population includes pre-treated patients with poor response after front-line chemotherapy. In this poor prognostic group, our results appear of interest with a median overall survival of 23.3 months, an objective response rate of $53.3 \%$, and complete response in $13.3 \%$ of cases.

Toxicity profile revealed a slightly high pulmonary toxicity. In general, the treatment with TKIs and concurrent radiation without chemotherapy showed a 4\% of G3-4 lung toxicity [12]. It is well known that adding chemotherapy to radiation causes an improvement in survival at the price of a higher toxicity. The grade 3 pneumonitis for patients treated with concurrent EGFR-TKI and chemoradiation is historically in the range of 6-8\% [11]. Reporting about the outcome of a phase I trial where gefitinib was administered concurrently with chemoradiation and used alone as maintenance therapy, G3-4 lung toxicity was $20 \%$ [12]. In our study the incidence of G3-5 lung toxicity is $8 \%$ and late pulmonary fibrosis was recorded only as G1-2 toxicity grade in $23 \%$

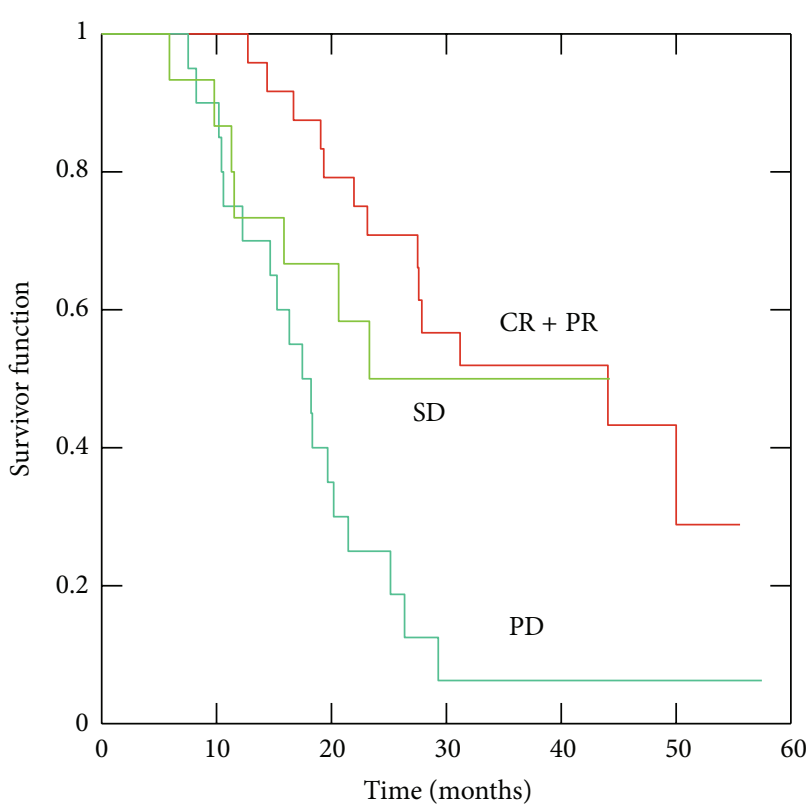

FIgURE 2: Overall survival according to clinical response after concurrent treatment with chemoradiation and TKIs therapy $(P=$ 0.001). Legend: CR: complete response; PR: partial response; SD: stable disease; PD: progression disease.

of patients; this is in line with toxicity levels of standard chemoradiation protocols. In recently reported phase III concomitant chemoradiation trials for locally advanced NSCLC, the overall survival ranged from 13.4 to 26.8 months. A meta-analysis showed 1- and 2-year survival rates of $57 \%$ and $45 \%$, respectively [3]. In our pre-treated cohort with locally advanced or metastatic NSCLC the median survival was 23.3 months with a 2 -year survival of $46 \%$. These interesting and surprising results confirmed Komaki et al. trial [14] where erlotinib was added to standard chemoradiation for stage III patients. They reported a very similar result to our, with a median survival of 25.8 months and 1-year OS 84\%.

Despite all the limitations of the observational nature of our study, which should be kept in mind when assigning clinical meaning to the evidence we report, adding TKIs to radiochemotherapy provided interesting results indeed. Over the next few years the clinical dilemma will be how to treat patients with locally advanced NSCLC and EGFR mutations: TKIs only, TKIs plus radiotherapy, or TKIs plus chemoradiation?

According to our experience, we can state that the addition of erlotinib to chemoradiation has a favorable safety profile and induces interestingly positive outcome. Further trials are needed to investigate the role of combined chemoradiation and TKIs or TKIs and radiation in untreated patients with EGFR mutations in order to assess the role of such treatment in locally advanced nonmetastatic NSCLC.

\section{Conflict of Interests}

Dr. G. Apolone received consulting fees from GSK, Italy, and GSK, UK. All other authors declare no conflict of interests. 


\section{References}

[1] F. Blackhall, M. Ranson, and N. Thatcher, "Where next for gefitinib in patients with lung cancer?" The Lancet Oncology, vol. 7, no. 6, pp. 499-507, 2006.

[2] F. A. Shepherd, J. R. Pereira, T. Ciuleanu et al., "Erlotinib in previously treated non-small-cell lung cancer," The New England Journal of Medicine, vol. 353, no. 2, pp. 123-132, 2005.

[3] A. Aupérin, C. Le Péchoux, E. Rolland et al., "Meta-analysis of concomitant versus sequential radiochemotherapy in locally advanced non-small-cell lung cancer," Journal of Clinical Oncology, vol. 28, pp. 2181-2190, 2010.

[4] T. Tanaka, A. Munshi, C. Brooks, J. Liu, M. L. Hobbs, and R. E. Meyn, "Gefitinib radiosensitizes non-small cell lung cancer cells by suppressing cellular DNA repair capacity," Clinical Cancer Research, vol. 14, no. 4, pp. 1266-1273, 2008.

[5] M. Baumann, M. Krause, E. Dikomey et al., "EGFR-targeted anti-cancer drugs in radiotherapy: preclinical evaluation of mechanisms," Radiotherapy and Oncology, vol. 83, no. 3, pp. 238-248, 2007.

[6] K. K. Ang, B. A. Berkey, X. Tu et al., "Impact of epidermal growth factor receptor expression on survival and pattern of relapse in patients with advanced head and neck carcinoma," Cancer Research, vol. 62, no. 24, pp. 7350-7356, 2002.

[7] R. K. Schmidt-Ullrich, R. B. Mikkelsen, P. Dent et al., "Radiation-induced proliferation of the human A431 squamous carcinoma cells is dependent on EGFR tyrosine phosphorylation," Oncogene, vol. 15, no. 10, pp. 1191-1197, 1997.

[8] P. Chinnaiyan, S. Huang, G. Vallabhaneni et al., "Mechanisms of enhanced radiation response following epidermal growth factor receptor signaling inhibition by erlotinib (Tarceva)," Cancer Research, vol. 65, no. 8, pp. 3328-3335, 2005.

[9] T. Ohnishi, T. Taki, S. Hiraga, N. Arita, and T. Morita, "In vitro and in vivo potentiation of radiosensitivity of malignant gliomas by antisense inhibition of the RAD51 gene," Biochemical and Biophysical Research Communications, vol. 245, no. 2, pp. 319-324, 1998.

[10] N. W. Choong, A. M. Mauer, D. J. Haraf et al., "Phase i trial of erlotinib-based multimodality therapy for inoperable stage III non-small cell lung cancer," Journal of Thoracic Oncology, vol. 3, no. 9, pp. 1003-1011, 2008.

[11] B. Center, W. J. Petty, D. Ayala et al., "A phase i study of gefitinib with concurrent dose-escalated weekly docetaxel and conformal three-dimensional thoracic radiation followed by consolidative docetaxel and maintenance gefitinib for patients with stage III non-small cell lung cancer," Journal of Thoracic Oncology, vol. 5, no. 1, pp. 69-74, 2010.

[12] J. Wang, T.-Y. Xia, Y.-J. Wang et al., "Prospective study of epidermal growth factor receptor tyrosine kinase inhibitors concurrent with individualized radiotherapy for patients with locally advanced or metastatic non-small-cell lung cancer," International Journal of Radiation Oncology Biology Physics, vol. 81, no. 3, pp. e59-e65, 2011.

[13] S. Ramella, L. Trodella, T. C. Mineo et al., "Beams arrangement in non-small cell lung cancer (NSCLC) according to PTV and dosimetric parameters predictive of pneumonitis," Medical Dosimetry, vol. 35, no. 3, pp. 169-178, 2010.

[14] R. Komaki, G. R. Blumenschein Jr., I. I. Wistuba et al., "Phase II trial of erlotinib and radiotherapy following chemoradiotherapy for patients with stage III NSCLC," Journal of Clinical Oncology, vol. 29, supplement, abstract 7020, 2011. 


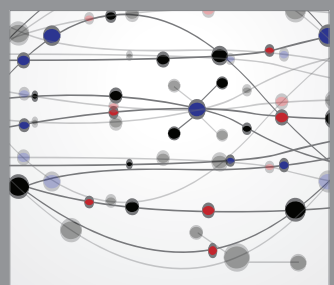

The Scientific World Journal
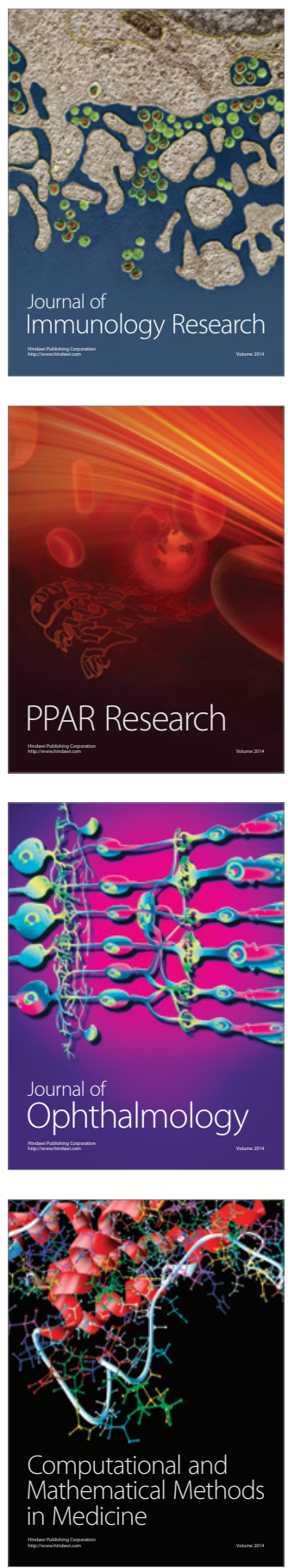

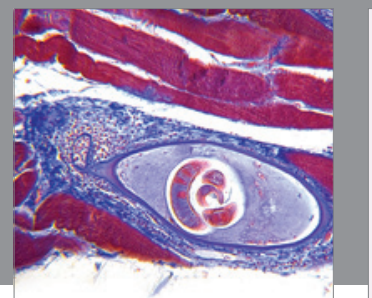

Gastroenterology

Research and Practice
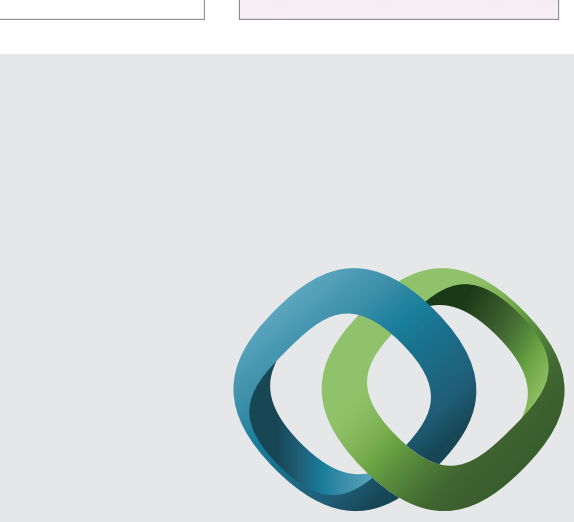

\section{Hindawi}

Submit your manuscripts at

http://www.hindawi.com
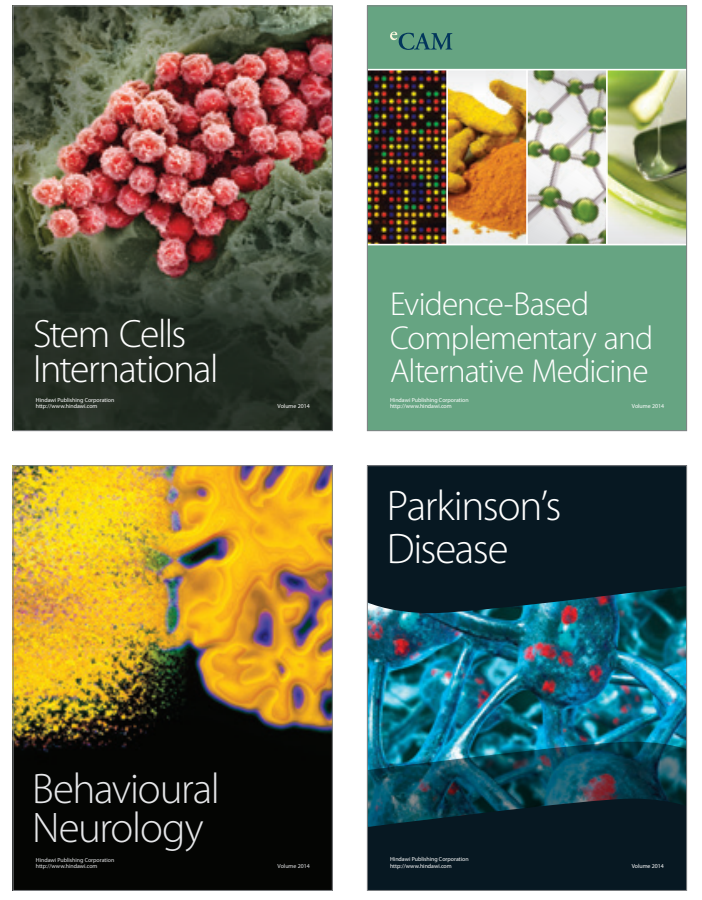
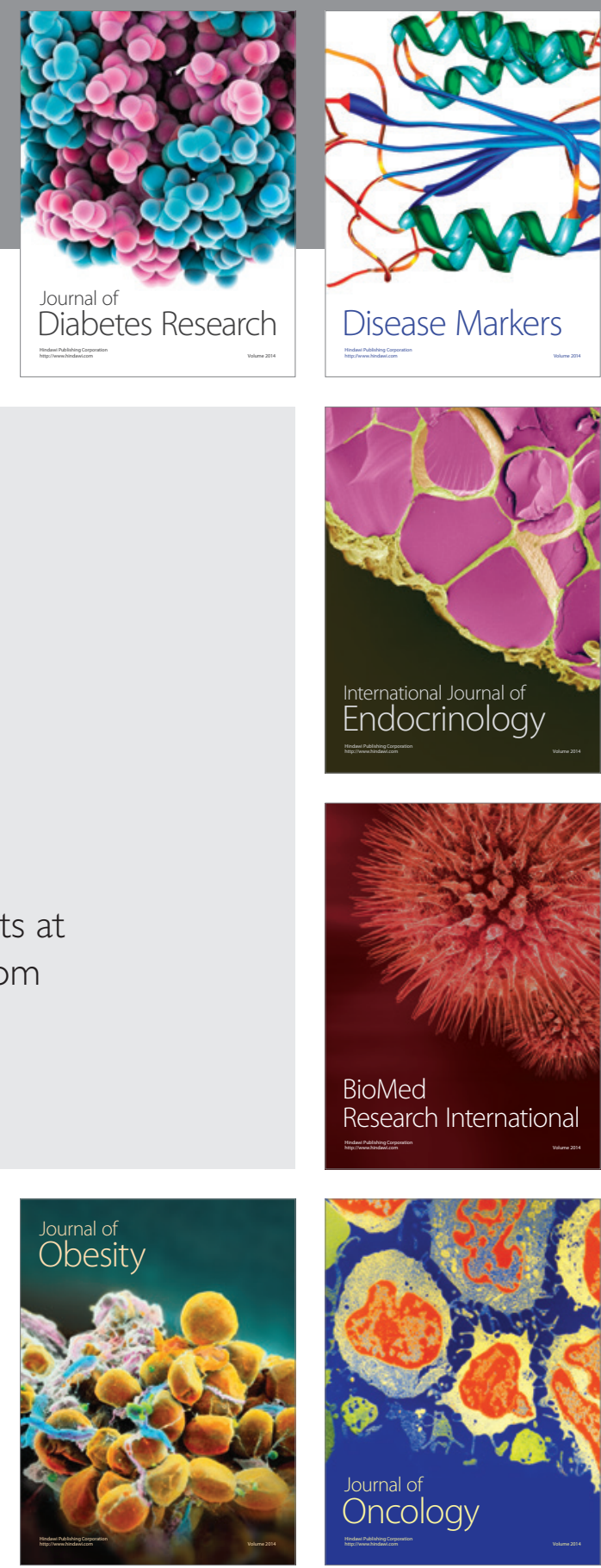

Disease Markers
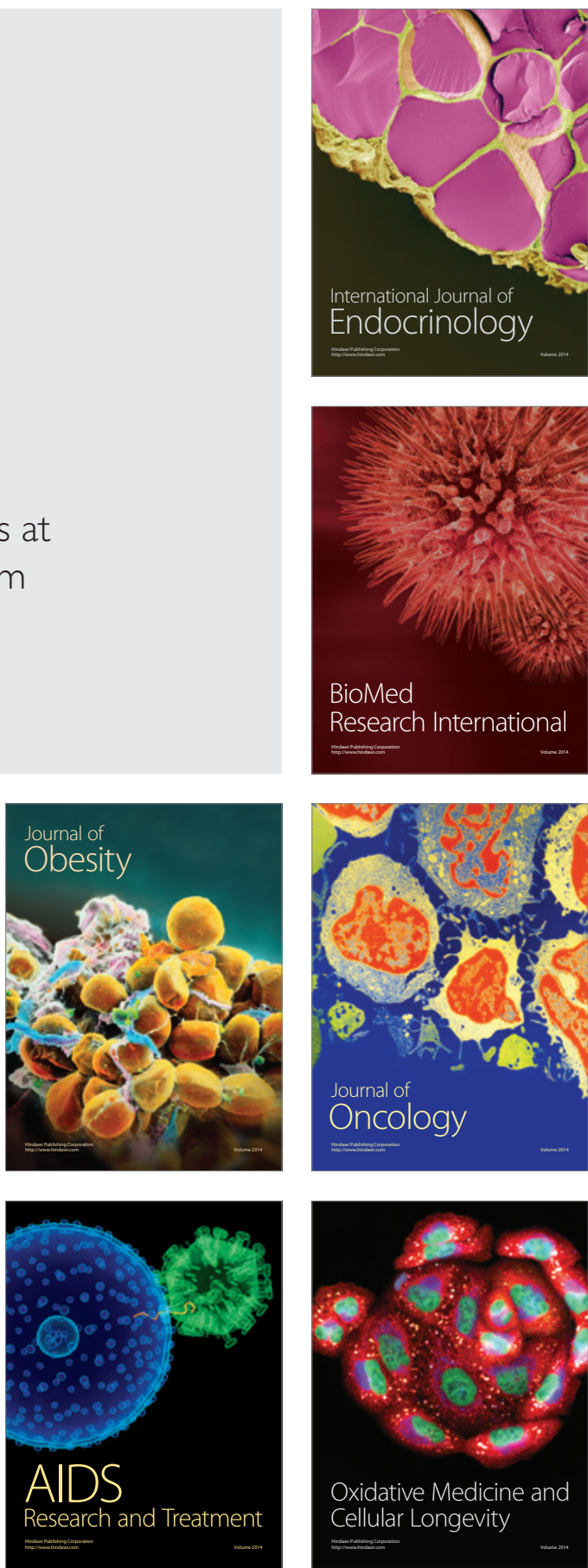